\title{
NSAID analgesic ketorolac used perioperatively may suppress early breast cancer relapse: particular relevance to triple negative subgroup
}

\section{Citation}

Retsky, Michael, Rick Rogers, Romano Demicheli, William J. M. Hrushesky, Isaac Gukas, Jayant S. Vaidya, Michael Baum, et al. Forthcoming. NSAID analgesic ketorolac used perioperatively may suppress early breast cancer relapse: particular relevance to triple negative subgroup. Breast Cancer Research and Treatment.

\section{Published Version}

doi:10.1007/s10549-012-2094-5

\section{Permanent link}

http://nrs.harvard.edu/urn-3:HUL.InstRepos:8923700

\section{Terms of Use}

This article was downloaded from Harvard University's DASH repository, and is made available under the terms and conditions applicable to Other Posted Material, as set forth at http:// nrs.harvard.edu/urn-3:HUL.InstRepos:dash.current.terms-of-use\#LAA

\section{Share Your Story}

The Harvard community has made this article openly available.

Please share how this access benefits you. Submit a story.

\section{Accessibility}


NSAID analgesic ketorolac used perioperatively may suppress early breast cancer relapse: particular relevance to triple negative subgroup

Michael Retsky, $\mathrm{PhD}^{1,5}$, Rick Rogers, $\mathrm{PhD}^{1}$, Romano Demicheli, MD, $\mathrm{PhD}^{2}$, William JM Hrushesky, $\mathrm{MD}^{3}$, Isaac Gukas, FMCS ${ }^{4}$, Jayant S Vaidya, FRCS ${ }^{8}$, Michael Baum, FRCS ${ }^{5}$, Patrice Forget, MD ${ }^{6}$, Marc DeKock, MD ${ }^{6}$, Katharina Pachmann, $\mathrm{MD}^{7}$

Breast Cancer Research and Treatment, 2012 May 24 [Epub ahead of print] PubMed PMID: 22622810.

The final publication is available at www.springerlink.com.

${ }^{1}$ Harvard School of Public Health, BLDG I, Rm 1311, 665 Huntington, Ave., Boston, MA 02115, USA.

${ }^{2}$ Department of Medical Oncology, Fondazione IRCCS Istituto Nazionale Tumori, Via Venezian 1, 20133 Milano, Italy. ${ }^{3}$ Oncology Analytics, Inc., 8751 W. Broward Blvd, Suite 500, Plantation, FL 33324. ${ }^{4}$ James Paget University Hospital, Lowestoft Road, Gorleston, Great Yarmouth, Norfolk, NR31 6LA. ${ }^{5}$ University College London, Royal Free and UCL Medical School, Centre for Clinical Science and Technology, Clerkenwell Building, Archway Campus, Highgate Hill, London, N19 5LW, UK; ${ }^{6}$ Universite catholique de Louvain, Department of Anesthesiology, St-Luc Hospital, av. Hippocrate 10-1821, 1200, Brussels, Belgium. ${ }^{7}$ Department of Experimental Hematology and Oncology, Clinic for Internal Medicine II, Friedrich Schiller University, Jena D-07747, Germany. ${ }^{8}$ Clinical Trials Group of the Division of Surgery and Interventional Science, University College London, Clerkenwell Building, Archway Campus, Highgate Hill, London N19 5LW, UK

Corresponding author: Michael Retsky phone 1-203-675-0017 email: michael.retsky@.gmail.com

\begin{abstract}
Background: To explain a bimodal relapse hazard among early stage breast cancer patients treated by mastectomy we postulated that relapses within 4 years of surgery resulted from something that happened at about the time of surgery to provoke sudden exits from dormant phases to active growth. Relapses at 10 months appeared to be surgery-induced angiogenesis of dormant avascular micrometastases. Another relapse mode with peak about 30 months corresponded to sudden growth from a single cell. Late relapses were not synchronized to surgery. This hypothesis could explain a wide variety of breast cancer observations. We have been looking for new data that might provide more insight concerning the various relapse modes.
\end{abstract}

Methods: Retrospective data reported in June 2010 study of 327 consecutive patients comparing various perioperative analgesics and anesthetics in one Belgian hospital and one surgeon. Patients were treated with mastectomy and conventional adjuvant therapy. Follow-up was average 27.3 months with range 13-44 months. Updated hazard as of September 2011 for this series is now presented.

Findings: NSAID ketorolac, a common analgesic used in surgery, is associated with far superior disease-free survival in the first few years after surgery. The expected prominent early relapse events are all but absent. In the 918 month period, there is 5-fold reduction in relapses. If this observation holds up to further scrutiny, it could mean that the simple use of this safe and effective anti-inflammatory agent at surgery might eliminate most early relapses.

Interpretation: Transient systemic inflammation accompanying surgery could be part of the metastatic tumor seeding process and could have been effectively blocked by perioperative anti-inflammatory agents. In addition, antiangiogenic properties of NSAIDs could also play a role. Triple negative breast cancer may be the ideal group with which to test perioperative ketorolac to prevent early relapses.

Funding: Susan Komen for the Cure

List of abbreviations used: NSAID: non steroid anti-inflammatory drug, CEBH: Commission d'Ethique Biomédicale Hospitalo-Facultaire de l'Université catholique de Louvain, CTC: circulating tumor cells, TNBC: triple negative breast cancer, IL-6: interleukin-6. 


\section{Background}

Carcinoma of the breast is one of the commonest causes of death among women in their middle age in the westernized countries of the world and increasing in incidence as the resource poor countries improve their GDP and start to emulate a "western" way of life. Clinicians continue to remain perplexed that the natural history and outcome of breast cancer remains unpredictable in spite of decades of work describing individual and composite prognostic factors and indices. We would argue that like the weather which used to be considered unpredictable, breast cancer can be better understood if one applies the correct mathematical model.

As previously reported, our analysis of data from the Milan National Cancer Institute found an unexpected bimodal pattern of relapse hazard among 1173 early stage breast cancer patients treated by mastectomy [1]. Figure 1 shows Milan data for premenopausal patients and fig. 2 shows postmenopausal patients in relapse hazard format.

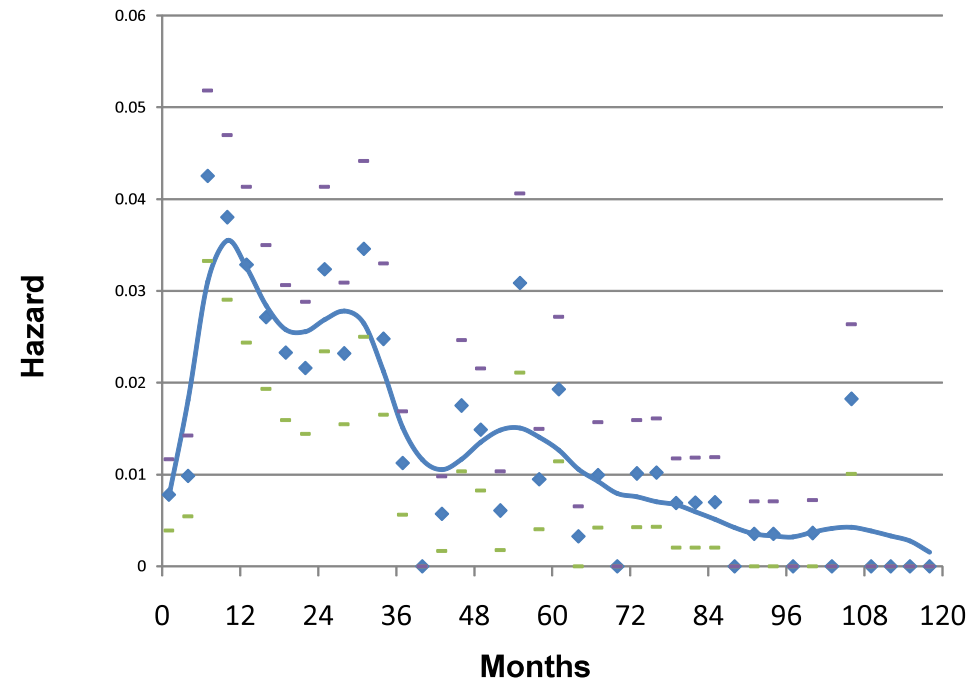

Fig 1 Hazard of relapse for premenopausal patients treated at Istituto Nazionale Tumori in Milan, Italy. Hazard is the number of events that occur in a time interval divided by the number of patients who enter that time as event free. Patients were treated by mastectomy well before the routine use of adjuvant therapy. The time interval in all hazard figures used here is 3 months. Average and standard deviations are indicated as diamonds and bars. The curve was obtained by a kernel-like smoothing procedure 


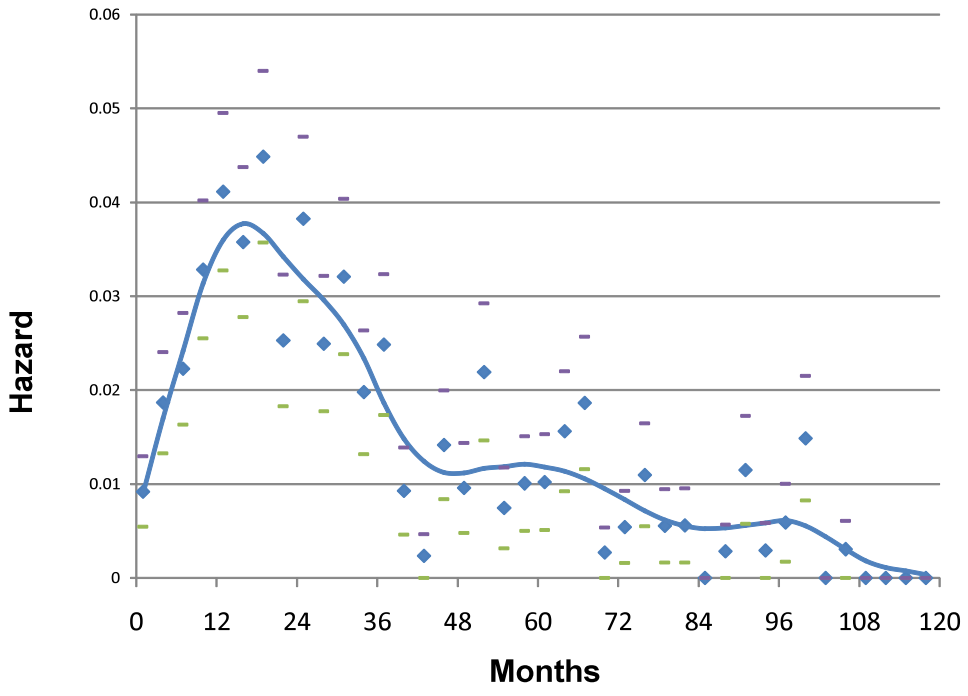

Fig 2 Same as fig. 1 except that these are the postmenopausal patients

There is an early peak of recurrence risk during the first three years of follow-up, a nadir at 50 months and a broad second peak extending from 60 months to over 15 years. Fifty to eighty percent of relapses, the proportion increasing with primary tumor size, reside within the first peak. Under closer examination, the first peak consists of two distinct groups centered at 10 months and 30 months that are well distinguishable in premenopausal patients but occur for postmenopausal patients as well. This pattern was not explainable by accepted theories.

Based on computer simulation, to explain the 10 month peak we postulated that induction of angiogenesis at the time of surgery provoked sudden exits from dormant avascular phases to active growth and then to detection. That mode is quite sharp and most often seen among premenopausal patients with positive nodes. We suggested the remainder of relapses within the first 48 months to be surgery-induced growth of previously dormant single malignant cells. We proposed that the broad late peak relapses result from steady stochastic progressions from single dormant malignant cells to avascular micro-metastases and then on to growing deposits.

We are certainly not the first to notice that removing breast tumors can sometimes accelerate disease and that this increases with tumor size. In fact, it was apparently known by Celsus (30BC - 38AD) and Galen (131 - 203AD). Remarkably, surgeons 2000 years ago were able to remove breast tumors and many patients survived the surgery. Aulus Cornelius Celsus wrote: "First there is the cacoethes, then carcinoma without ulceration, then the fungating ulcer. None of these can be removed but the cacoethes: the rest are irritated by every method of cure. The more violent the operations the more angry they grow. Some use caustics, some burning irons, others remove the growth with the scalpel. After excision, even though a cicatrix is formed, it recurs, bringing with it the cause of death, whereas at the same time, most people, by using no violent methods to attempt the extirpation of the disease but only applying mild medications to soothe it, protract their lives, notwithstanding the disorder, to an extreme old age." This is of course relative to the life expectancy in those times. Galen wrote: "We have often cured this disease in the early stages, but after it has grown to a noticeable size no one has cured it with surgery" [2].

Computer simulations of the two early relapse modes are shown in fig. 3. Using these data and analyses, we have been able to help explain a wide variety of previously unpredictable breast cancer observations. These include the high effectiveness of adjuvant chemotherapy seen in premenopausal node positive women and why mammographic screening is more effective for women age 50-59 than for women age 40-49. 


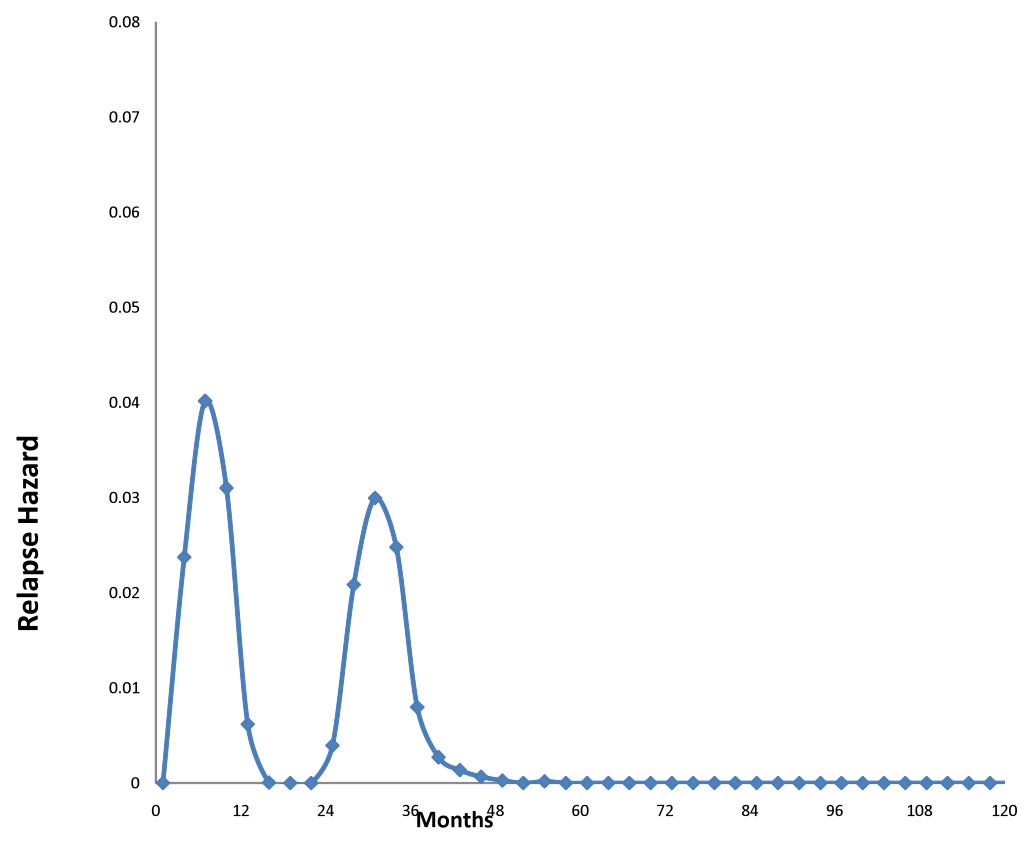

Fig. 3 Computer simulations of early relapse events. Hazard of relapse for early events centered at 10 months and at 30 months post surgery as proposed by computer simulation. Simulations included effects of mastectomy and were based on Milan data shown in figs. 1 and 2. The 10 month and 30 month events may be distinguished in fig. 1 and less clear but present in fig. 2

The proposed model was further supported by the fact that in patients given adjuvant chemotherapy targeting proliferating cells first year (angiogenesis switching-related) recurrences and third year (single cell wake up-related) recurrences were remarkably reduced. Indeed, additional data from Milan for patients treated with CMF adjuvant chemotherapy (fig. 4) provided evidence that the recurrence risk pattern of patients receiving adjuvant chemotherapy displays a single initial peak at 18-20 months and a late peak at 60 months. Similar patterns have now been identified in 21 independent databases from US, Europe and Asia.

The most important finding of this early work is that something happens at or about the time of surgery to accelerate or induce metastatic activity that results in early relapses. These early relapses comprise over half of all relapses. Surgery-induced angiogenesis of dormant avascular micrometastases and surgery-induced activity of single malignant cells are implicated. Late relapses are apparently not synchronized to the time of surgery. We have been vigilantly looking for new data with which we can learn more about surgery-induced tumor activity and that perhaps will also lead to improved outcomes. As we report here, there has been an important development.

\section{Methods}

In June 2010, Forget et al reported data from a retrospective disease free survival study of 327 consecutive patients comparing various perioperative analgesics and anesthetics (sufentanil, clonidine, ketorolac, and ketamine) in one Belgian hospital and one surgeon [3].

Approval of the Ethical Committee of St-Luc Hospital was provided by the CEBH of the Université Catholique de Louvain (Brussels, Belgium), Chairperson Prof Dr. J.M. Maloteaux. Investigators were unable to obtain consent from the patients for this retrospective study and the need for written informed consent from participants was waived, as accepted by the CEBH. 


\section{$N+(1-3)$}

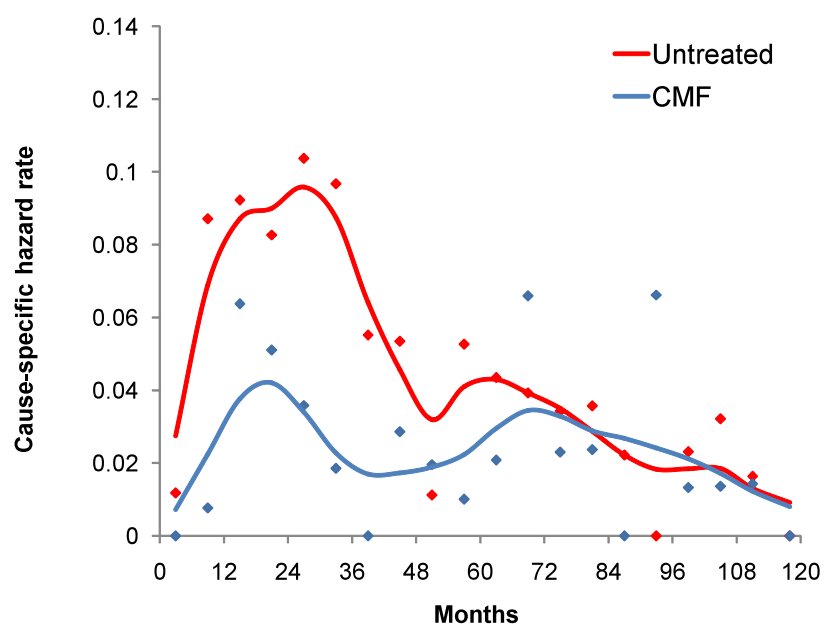

Premenopausal plus postmenopausal patients

Fig. 4 The result of adjuvant CMF chemotherapy. The two early peaks in the untreated population coalesce into a single peak in this treated population at about 20 months. Apparently CMF chemotherapy acts to produce most extensive reduction in relapse hazard in the $1^{\text {st }}$ and $3^{\text {rd }}$ year

Patients were treated with mastectomy and conventional adjuvant therapy. Chemotherapy, radiotherapy, and endocrine therapy were performed according to international expert consensus protocols (9th and 10th St-Gallen consensus). Follow-up in that initial report was average 27.3 months with range 13-44 months. Patients who received anti-inflammatory drugs were compared with those who had not and their hazard of recurrence was analyzed and compared. We now report an independent update of those data as of September 2011.

\section{Findings}

Perioperative administration of the NSAID ketorolac, a common surgical anti-inflammatory analgesic, was associated with significantly superior disease-free survival in the first few years after surgery. The expected prominent early relapse risk peak is all but absent in the 2010 ketorolac data (fig. 5). The few events in the ketorolac group show a small bump in the first 10 months and then slowly rising until the $4^{\text {th }}$ year when follow-up of this series ends. After 24 months the ketorolac group hazard rate pattern is indistinguishable from the corresponding pattern for the no-ketorolac group. The updated analysis presented in fig. 6 shows the benefit appears in the 9-18 month hazards and is of magnitude $4-6$ fold, consistent with the early report. Specifically in that 9 month period there are 3 relapses in the ketorolac group compared to 15 in the no-ketorolac patients.

\section{Interpretation}

Even with the insight of simulations, it is sometimes impossible to determine with certainty what happened to each of the various relapse modes in a particular report. However in this case it appears that perisurgical ketorolac is associated with a dramatic reduction of the recurrences that, according to the proposed model, are related to the surgery-induced metastatic activity. If this observation holds up to further scrutiny, it could mean that the simple use of this safe and effective anti-inflammatory agent at the time of surgery might eliminate most early relapses.

Published along with the original Forget et al study, an outline of a number of possible effects of surgery and anesthesia on cancer growth was presented by Gottschalk et al [4]. These include stress, immunosuppression, pain, 


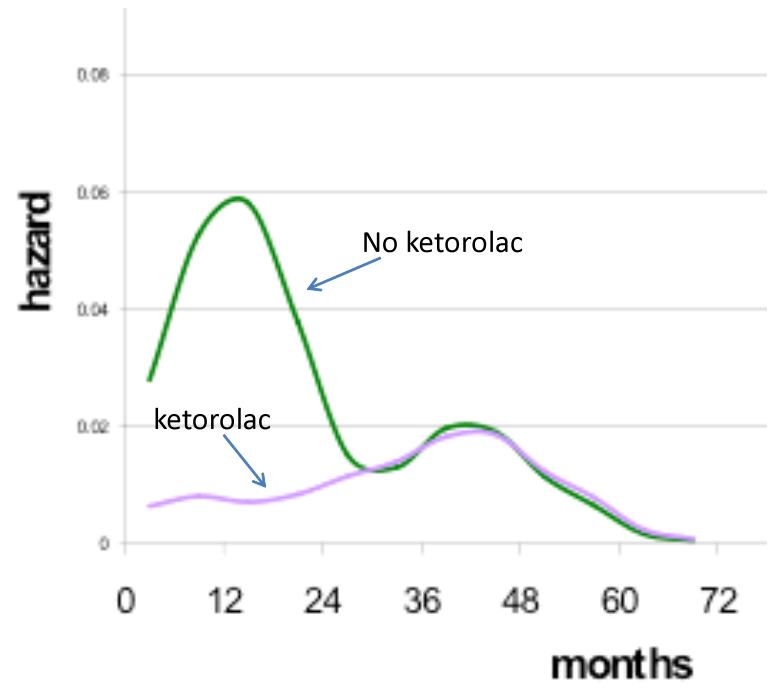

Fig. 5 Forget et al [3] data from Universite catholique de Louvain in Brussels, Belgium. Relapse hazard is shown for mastectomy patients given ketorolac or not. Data are smoothed as indicated for fig. 1. In the months 9 through 18, there were 3 relapse events in the ketorolac group compared to 15 in the no-ketorolac group

transfusion, inflammation, hypothermia, and a few others. In view of the extensive literature discussing connections and correlations between cancer growth and inflammation, our interest was drawn toward inflammation as possibly a key metastasis producing process.

Balkwill et al writes that if genetic damage is the "match that lights the fire" of cancer, then inflammation is the "fuel that feeds the flames" and that inflammation affects both the survival and proliferation of already initiated cancer cells [5]. Since Virchow first proposed in 1863 that tumors could originate from sites of chronic inflammation, it has been well established that chronic inflammation both contributes to cancer progression and predisposes tissue to various types of primary and metastatic cancer [6].

Based on Pascual et al data from a colon cancer study, transient inflammation can be both local and systemic [7]. They measured the proinflammatory cytokine interleukin-6 (IL-6) in serum prior to surgery and in peritoneal fluid during surgery to establish baseline IL-6, and again at 4, 12, 24 and 48 hours and at 4 days after surgery to determine a temporal trend. They found levels of IL- 6 in serum at approximately $1 / 300$ of the concentrations seen in peritoneal fluid. Judging by their data it would seem that levels in serum would gradually return to baseline in a week or so. While not breast cancer surgery, we can assume that systemically and transiently something similar occurs in surgery to remove breast cancer.

The severity, timing, and local character of any particular inflammatory response, initiated by tissue damage, depends on the cause, location and site of the area affected, and host's condition [8]. Inflammatory oncotaxis, a term used to describe tumor growth at a site of inflammation, is occasionally seen in persons with known or occult cancer and who have local trauma [9-12]. Martins-Green et al studied an avian system in which a virus is the carcinogenic agent [13]. When newly hatched chicks are given injections of Rous sarcoma virus, a tumor develops only at the site of injection unless a wound is made a distance away from the primary tumor where a tumor develops at the site of 


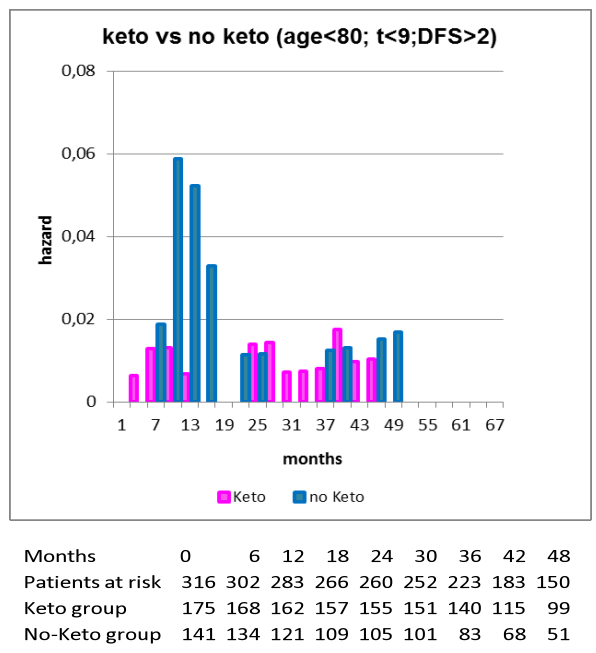

Fig. 6 Forget et al data were updated September 2011 and shown in hazard form but not smoothed as in fig. 5. Patient data are presented in the table. Patients included in this figure were less than 80 years of age, tumor less than $9 \mathrm{~cm}$ diameter and disease free survival greater than 2 months. It can be seen that relapses in months $9-18$ accounted for the major difference between ketorolac and non-ketorolac patients.

wounding. They found that when inflammation was inhibited, tumors were also inhibited; when inflammation could not be stopped, tumors developed as before.

Among different possible reasons underlying this phenomenon, perhaps the simplest is more or less mechanical. It is well established that many cancer patients have circulating tumor cells $[14,15]$. Blood flow in capillaries is only $0.03 \mathrm{~cm} / \mathrm{sec}$ which would make leaky capillary venules a very efficient way for circulating tumor cells to enter tissue. Perhaps the transient systemic inflammation accompanying surgery and subsequent inflammatory oncotaxis is part of the metastatic tumor seeding process. It may be that what we previously called dormant single cells induced into metastatic growth were at least in some cases residing not at the site of eventual relapse. Rather, circulating tumor cells in an inflammatory environment extravasate, resulting months later in a metastatic tumor. Circulating tumor cells are a reality. Surgical induction of inflammation is universal. Capillary leakage is enhanced by inflammation. It is thereby logical to expect that an effective perisurgical anti-inflammatory strategy may affect surgery-induced and possibly angiogenesis-mediated cancer spread.

In addition, the hypothesis that decreasing the inflammatory response to the surgical maneuver could interfere with the angiogenesis switch of avascular micrometastases is not outlandish. A few hypotheses can be put forward, including, but not limited to, the following.

At steady state conditions in adult mammals, most endothelial cells are quiescent and are believed to contribute to organ homeostasis and tumor dormancy [16]. However, in response to inflammation the upregulation and release of factors stimulating endothelial cells to proliferate could also induce endothelial cells to secrete specific cytokines that reciprocally support the regeneration of normal and malignant stem cells. In this regard, activation of the so called "vascular niche" will stimulate tumor growth [17]. Therefore, the angiogenesis switch would result from the interaction of tumor cells and the vascular niche influenced by surgery, resulting in the dormancy interruption. If so, reducing inflammation could result in impairment of the dormant foci wake up process [18]. 


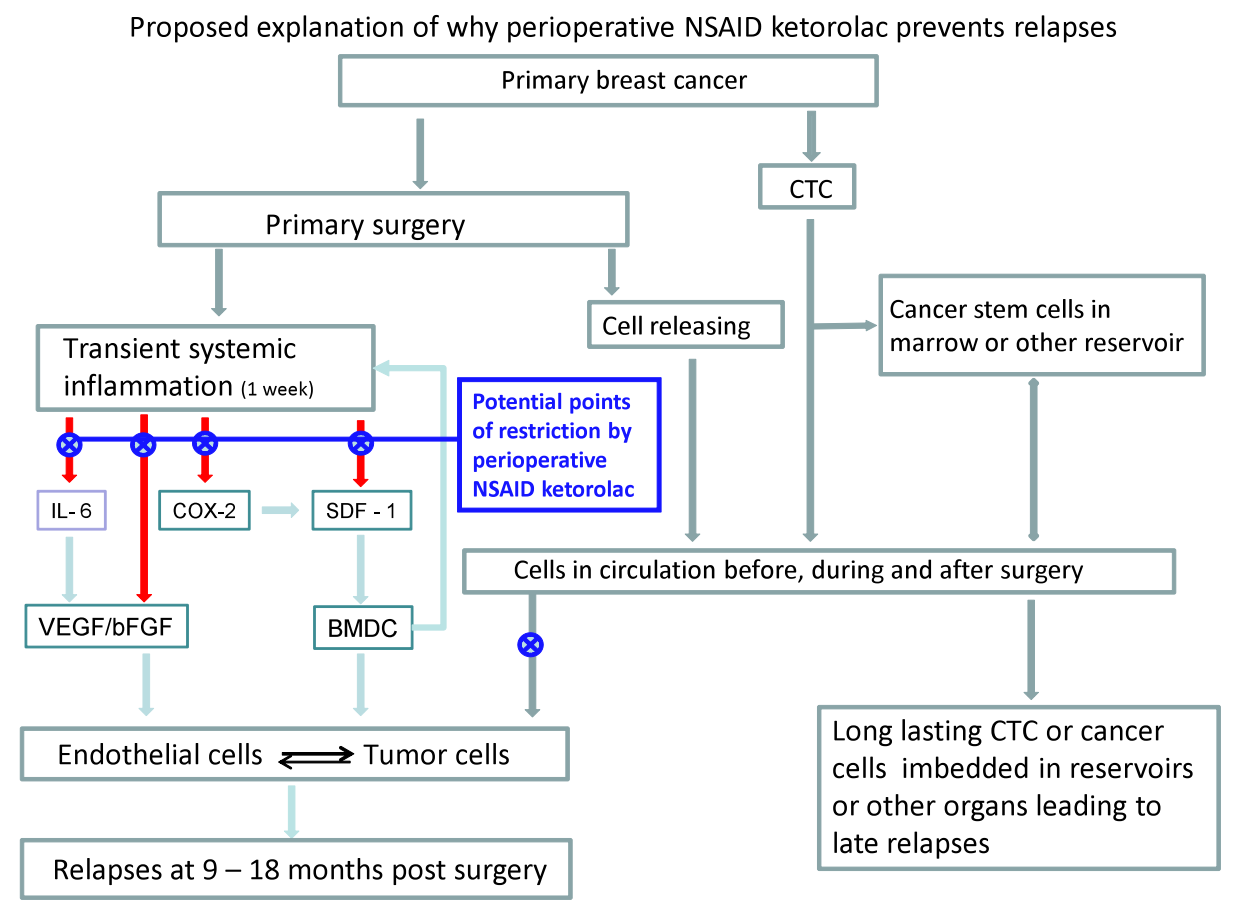

Fig 7 Symbolic description of proposed explanations for Forget et al data. Early relapses are assumed to be related, at least in part, to the inflammatory process due to primary tumor surgical removal, directly or indirectly eliciting peritumoral endothelial cell proliferation. A few possible mechanisms are explained. A) Angiogenic factors, like VEGF and bFGF, are directly released or even produced via IL-6; B) Bone marrow derived CXCR-4 positive cells, acting both on tumor foci and on the inflammatory process, are mobilized by SDF-1 directly released or even produced via COX-2. Perioperative Ketorolac would restrict both endocrine and cellular pathways, thus impairing the metastatic process. CTC refers to circulating tumor cells.

Tissue lesions induce mobilization of bone marrow derived cells that are capable of responding to chemo-attractant signals from various organs, where they undergo a homing process and where they release several chemokines [19]. This phenomenon is prominent during neovascularization of wounded tissues via direct or paracrine activity inducing capillary formation. Bone marrow derived cells may play an important role in tumor angiogenesis [20]. Reducing inflammation induced by primary tumor surgical removal could interfere with these processes and thus with metastasis development.

A common basis of the above mentioned processes is cell trafficking, a main regulator of which is the SDF1CXCR4 axis that is also involved in tumor cell trafficking [19]. NSAIDs may interfere with SDF1 levels via the pathway COX-2 $\rightarrow$ PGE $\rightarrow$ SDF-1, thus resulting in impairment of processes underlying metastasis development.

It cannot be excluded that all the above mentioned machineries could act together. It has been recently reported in an animal model, where mice with s.c. implantation of Lewis Lung Carcinoma were subjected to an operative injury, that surgery induced the release of cytokines/chemokines and mobilized bone marrow-derived cells [21]. These mobilized cells were then recruited into tumor tissue with concomitant enhancement of angiogenesis, thereby accelerating tumor growth. Furthermore, blocking recruitment of bone marrow stem cells by disrupting SDF/CXCR signals completely negated the accelerated tumor growth.

Lastly, it cannot be ruled out that the reduced recurrence risk for patients receiving ketorolac may be attributed, at least in part, to the reduced usage of opioids for pain management with ketorolac but more likely its antiangiogenic properties [22-24]. 


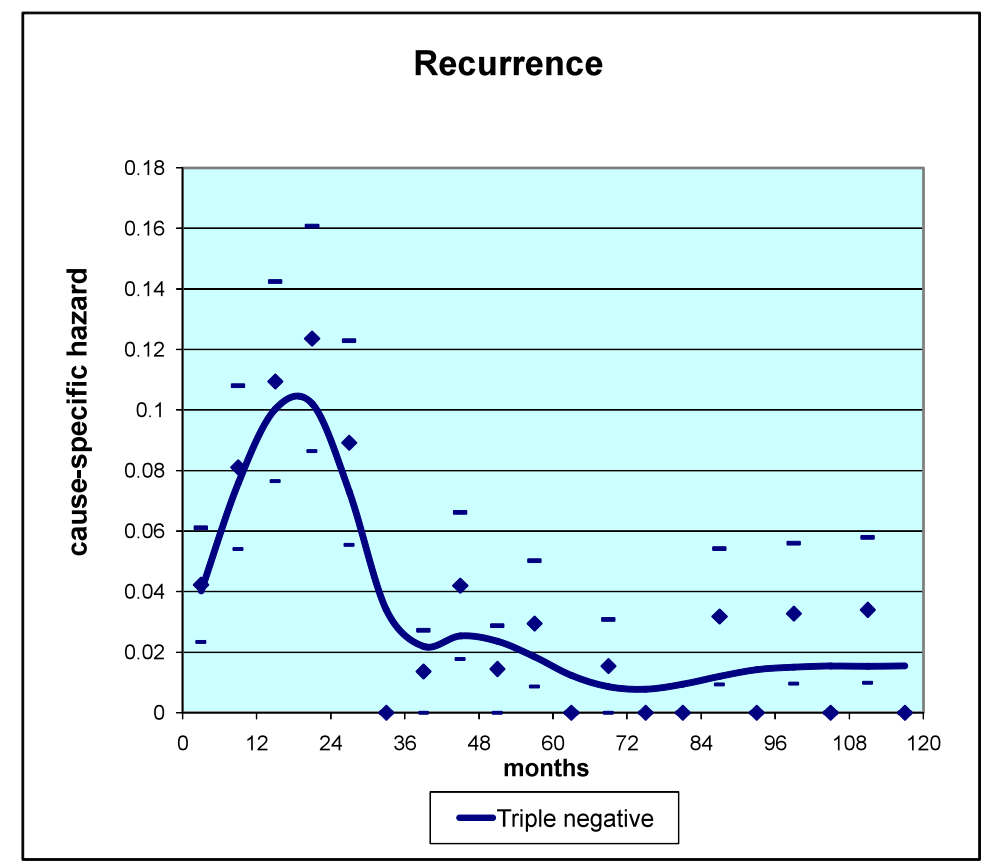

Fig. 8 Hazard data from a Milan database for 121 TNBC patients with 10-11 years follow up. There are 50 relapse events within 5 years of surgery. The general similarity of these hazard data to Forget et al data for no-ketorolac patients leads to the suggestion that TNBC may be the ideal study group with which to test perioperative ketorolac

A more indirect effect of ketorolac on metastasis development might be hypothesized when circulating tumor cells are considered. Data from Pachmann show a surge in circulating epithelial cells after primary breast cancer surgery, but intriguingly, that surge occurs 3-7 days after surgery [25]. Such a delayed increase in what may be circulating tumor cells after breast cancer surgery was also reported by Daskalakis et al [26]. This phenomenon recalls the surge of bone marrow derived cells elicited by tissue damage (e.g., myocardial infarction) [27]. It further suggests that tumors may share physiological mechanisms with normal tissues and, moreover, that inhibiting the inflammatory process might reduce late metastases as well, an occurrence not evidenced by the Forget et al study but might be seen in a recent report of daily use of aspirin [28].

Figure 7 shows a schematic description of what we suspect to be the mechanisms governing metastatic relapse from early breast cancer. Are the missing early relapses never to happen or are they merely postponed to become late relapses? Whatever their source and shedding timing, cancer cells in circulation may have half life of a few days or less. Unless injected into more hospitable surroundings such as tissue, these cells will likely harmlessly die off. These data and our analysis suggest that at least for some patients the early relapses apparently avoided in the Forget et al data do not show up later.

We now turn our attention to methods of testing this new hypothesis. Animal studies would be very important, however we think this should be tested prospectively in a clinical trial. The next question that arises is what patient group would be a good candidate for a trial. Most breast cancer clinical trials, at least in the US, focus on distinct patient subgroups based on recurrence risk levels. The triple negative subgroup attracted our attention for several reasons. Lacking markers that strongly suggest that there is benefit of targeted therapy (HER2, ER, PgR), triple negative breast cancer (TNBC) is looked upon by clinicians as a "bad tumor" with high recurrence rate in spite of adjuvant chemotherapy. That pessimistic viewpoint seems justified since TNBC has approximately $10 \%$ incidence but accounts for $20 \%$ of mortality in breast cancer. 
We had access to a TNBC data base from Milan that we analyzed with our hazard methods. The relapse hazard (fig. 8) looks remarkably similar to the no-ketorolac group in the Forget et al study reported above and shown in fig. 5. Triple negative breast cancer therefore appears to be the ideal study group with which to test benefit of perioperative ketorolac in a clinical trial.

As noted by Wallace et al, the racial disparity in breast cancer outcome is due primarily to deaths within the first few years after diagnosis providing an additional motivation to test at the earliest opportunity what we report here [29].

\section{Summary and Conclusions}

Our previous findings were that most relapses occurring within 4 years may be induced by the effects of breast cancer surgery. We now report that perioperative non-steroid anti-inflammatory agents appear to abrogate the early hazard of recurrence.

This suggests at least some of the early relapse events are related to transient systemic inflammation that may involve the switch of avascular micrometastases to neo-angiogenesis via a number of possible mechanisms, a few of which are outlined above. "Something for nothing" rarely if ever happens in cancer therapy. High priority should be given to test this hypothesis in a randomized trial as it is implementable regardless of state of socio-economic development because expensive drugs, modern imaging facilities and advanced pathology services are not particularly relevant to implementing this simple change.

Such verification of the Forget et al findings could be relatively simple to pursue by piggy-backing on another randomized trial in early stage breast cancer, in particular addressing treatments for TNBC. In the retrospective analysis by Forget et al, the differences in relapse hazard are strong and occur within the first two years after surgery so we should find out in a relatively short time if these data are confirmed.

While there is much worthwhile interest in personalized cancer treatment, that may not be the only way to solve the early relapse problem nor would it ever be affordable in the resource poor parts of the globe.

Competing interests: Michael Retsky has a patent pending for treatment of early stage cancer and is on the Board of Directors of the Colon Cancer Alliance (www.ccalliance.org). Authors declare no other competing interests.

Authors' contributions: $\mathrm{PF}$ and $\mathrm{MDeK}$ conceived the retrospective analgesic study and provided the Brussels data; RD provided the Milan data and hazard analysis; MR did the computer simulation and drafted the manuscript; WJMH, RD and JSV participated in writing the manuscript; MR, RD, MB, IG, RR, WJMH, and JSV participated in discussions leading to the surgery-transient systemic inflammation-inflammatory oncotaxis hypothesis. KP provided information on temporal trends of circulating epithelial cells after mastectomy. All authors made substantive intellectual contributions to the study and participated in preparing and approving the manuscript. The corresponding author had full access to all the data reported in the study and had final responsibility to submit for publication.

Acknowledgements: This work is supported in part by grant 100484 from Susan G Komen for the Cure. The funding organization played no role in design of study, collection and interpretation of data, decision to publish or writing of paper. Brussels data updated September 2011 shown in fig. 6 were provided by Sarah Aram and analyzed by Romano Demicheli.

\section{References}

1. Retsky M, Demicheli R, Hrushesky W, Baum M, Gukas I (2010) Surgery Triggers Outgrowth of Latent Distant Disease in Breast Cancer: An Inconvenient Truth? Cancers 2:305-337. http://www.mdpi.com/2072$\underline{6694 / 2 / 2 / 305 /}$

2. Irving Ariel (1994) A historical review of breast cancer treatment. In: Houston Johnson and Leslie Wise, eds. Breast Cancer: Controversies in Management, Armonk, NY, Futura. 
3. Forget P, Vandenhende J, Berliere M, Machiels JP, Nussbaum B, Legrand C, De Kock M 2010) Do intraoperative analgesics influence breast cancer recurrence after mastectomy? A retrospective analysis. Anesth Analg Jun 1, 110(6):1630-5. http://www.ncbi.nlm.nih.gov/pubmed/20435950

4. Gottschalk A, Sharma S, Ford J, Durieux ME, Tiouririne M (2010) Review article: the role of the perioperative period in recurrence after cancer surgery. Anesth Analg Jun 1;110(6):1636-43. Epub 2010 Apr 30. PubMed PMID: 20435944.

5. Balkwill F, Mantovani A (2001) Inflammation and cancer: back to Virchow? Lancet 0099-5355, Feb 17 357: 9255 .

6. Keibel A, Singh V, Sharma MC (2009) Inflammation, microenvironment, and the immune system in cancer progression. Curr Pharm Des 15(17):1949-55. Review.

7. Pascual M, Alonso S, Parés D, Courtier R, Gil MJ, Grande L, Pera M (2011) Randomized clinical trial comparing inflammatory and angiogenic response after open versus laparoscopic curative resection for colonic cancer. Br J Surg Jan, 98(1):50-9.

8. Demaria S, Pikarsky E, Karin M, Coussens LM, Chen YC, El-Omar EM, Trinchieri G, Dubinett SM, Mao JT, Szabo E, Krieg A, Weiner GJ, Fox BA, Coukos G, Wang E,Abraham RT, Carbone M, Lotze MT (2010) Cancer and inflammation: promise for biologic therapy. J Immunother May, 33(4):335-51. Review.

9. Jones FS, Rous P (1914) On The Cause Of The Localization Of Secondary Tumors At Points Of Injury. $J$ Exp Med Oct 1, 20(4):404-12.

10. DerHagopian RP, Sugarbaker EV, Ketcham A (1978) Inflammatory oncotaxis. JAMA Jul 28;240(4):374-5. PubMed PMID: 660874.

11. El Saghir NS, Elhajj II, Geara FB, Hourani MH (2005)Trauma-associated growth of suspected dormant micrometastasis. BMC Cancer Aug 4, 5:94.

12. Walter ND, Rice PL, Redente EF, Kauvar EF, Lemond L, Aly T, Wanebo K, Chan ED (2011) Wound healing after trauma may predispose to lung cancer metastasis: review of potential mechanisms. Am J Respir Cell Mol Biol May, 44(5):591-6. Epub 2010 Dec 22. Review.

13. Martins-Green M, Boudreau N, Bissell MJ (1994) Inflammation is responsible for the development of wound-induced tumors in chickens infected with Rous sarcoma virus. Cancer Res Aug 15, 54(16):4334-41.

14. Krebs MG, Hou JM, Ward TH, Blackhall FH, Dive C (2010) Circulating tumour cells: their utility in cancer management and predicting outcomes. Ther Adv Med Oncol Nov, 2(6):351-65.

15. Zhe X, Cher ML, Bonfil RD (2011) Circulating tumor cells: finding the needle in the haystack. Am J Can Res 1(6):740-51.

16. Carmeliet P, Jain RK (2000) Angiogenesis in cancer and other tissues. Nature 407:249-257.

17. Butler JM, Kobayashi H, Rafii S (2010) Instructive role of the vascular niche in promoting tumor growth and tissue repair by angiocrine factors. Nat Rev Cancer 10:138-146.

18. Favaro E, Amadori A, Indraccolo S (2008) Cellular interaction in the vascular niche: implications in the regulation of tumor dormancy. APMIS 116:648-659.

19. Kavanagh DP, Kalia N (2011) Hematopoietic stem cell homing to injured tissues. Stem Cell Rev 7:672-682. 
20. Laurent J, Touvrey C, Botta F, Kuonen F, Ruegg C (2011) Emerging paradigms and questions on proangiogenic bone marrow-derived myelomonocytic cells. Int J Dev Biol 55(4-5):527-34.

21. Takemoto Y, Li TS, Kubo M, Ohshima M, Ueda K, Harada E, Enoki T, Okamoto M, Mizukami Y, Murata T, Hamano K (2011) Operative injury accelerates tumor growth by inducing mobilization and recruitment of bone marrow-derived stem cells. Surgery Jun;149(6):792-800. Epub 2011 Apr 20 PubMed PMID: 21507448.

22. Bortsov AV, Millikan RC, Belfer I, Boortz-Marx RL, Arora H, McLean SA (2012) $\mu$-Opioid Receptor Gene A118G Polymorphism Predicts Survival in Patients with Breast Cancer. Anesthesiology Apr;116(4):896-902. PubMed PMID: 22433205; PubMed Central PMCID: PMC3310356.

23. Pakneshan P, Birsner AE, Adini I, Becker CM, D'Amato RJ (2008) Differential suppression of vascular permeability and corneal angiogenesis by nonsteroidal anti-inflammatory drugs. Invest Ophthalmol Vis Sci Sep, 49(9):3909-13.

24. Burke JP, Pestotnik SL, Classen DC, Lloyd JF (1996) Evaluation of the financial impact of ketorolac tromethamine therapy in hospitalized patients. Clin Ther Jan-Feb;18(1):197-211. PubMed PMID: 8851463

25. Pachmann K (2011) Tumor Cell Seeding During Surgery-Possible Contribution to Metastasis Formations. Cancers 3:2540-2553.

26. Daskalakis M, Mavroudis D, Sanidas E, Apostolaki S, Askoxylakis I, de Bree E, Georgoulias V, Melissas J (2011) Assessment of the effect of surgery on the kinetics of circulating tumour cells in patients with operable breast cancer based on cytokeratin-19 mRNA detection. Eur J Surg Oncol May, 37(5):404-10. Epub 2011 Mar 4.

27. GrØgaard HK, Solheim S, Landsverk KS, Seljeflot I, Hoffmann P, Arnesen H, Ilebekk A (2011)

Circulating CD£\$+ progenitor cells and growth factors in patients treated with PCI for acute myocardial infarction or stable angina pectoris. Scand J Clin Lab Invest 71:322-329.

28. Rothwell PM, Price JF, Fowkes FG, Zanchetti A, Roncaglioni MC, Tognoni G, Lee R, Belch JF, Wilson M, Mehta Z, Meade TW (2012). Short-term effects of daily aspirin on cancer incidence, mortality, and non-vascular death: analysis of the time course of risks and benefits in 51 randomised controlled trials. Lancet. $2012 \mathrm{Apr} 28$; 379(9826): 1602-12. Epub Mar 21. PubMed PMID: 22440946.

29. Wallace TA, Martin DN, Ambs S (2011) Interactions among genes, tumor biology and the environment in cancer health disparities: examining the evidence on a national and global scale. Carcinogenesis Aug, 32(8):110721. 